\title{
THE CAUCHY PROBLEM FOR DOUGLIS-NIRENBERG ELLIPTIC SYSTEMS OF PARTIAL DIFFERENTIAL EQUATIONS(1) \\ BY
}

\author{
RICHARD J. KRAMER
}

\begin{abstract}
Several partial answers are given to the question: Suppose $U$ is a solution of the Douglis-Nirenberg elliptic system $L U=F$ where $F$ is analytic and $L$ has analytic coefficients. If $U=0$ in some appropriate sense on a hyperplane (or any analytic hypersurface) must $U$ vanish identically?

One answer follows from introducing a so-called formal Cauchy problem for Douglis-Nirenberg elliptic systems and establishing existence and uniqueness theorems.
\end{abstract}

A second Cauchy problem, in some sense a more natural one, is discussed for an important subclass of the Douglis-Nirenberg elliptic systems. The results in this case give a second partial answer to the original question.

The methods of proof employed are largely algebraic. The systems are reduced to systems to which the Cauchy-Kowalewski theorem applies.

1. Introduction. We formulate and solve several Cauchy problems for systems of partial differential equations which are elliptic in the sense of Douglis and Nirenberg [4, pp. 504ff.]. These Cauchy problems arise from attempts to answer the following question about solutions of the elliptic systems of Douglis-Nirenberg.

Question. Must a solution $U$ of the homogeneous generalized elliptic system $L U=0$ which vanishes identically (to some appropriate order) on a hyperplane be identically zero?

For a single elliptic operator with analytic coefficients the answer is given in the affirmative by the Cauchy-Kowalewski theorem and the Holmgren uniqueness theorem. The result extends immediately to standard elliptic systems but not to Douglis-Nirenberg elliptic systems. To see this and to fix ideas, one should keep in mind the following example. Write the Laplacian $D_{x}^{2} u+D_{y}^{2} u=0$, where $D_{x}=$ $\partial / \partial x, D_{y}=\partial / \partial y$ as a first order system by introducing new unknown functions $u^{1}, u^{2}, u^{3}$ and setting $u=u^{1}, D_{x} u=u^{2}, D_{y} u=u^{3}$ to obta in

Received by the editors October 12, 1972.

AMS (MOS) subject classifications (1970). Primary 65M30.

Key words and phrases. Cauchy problem, formal Cauchy data, Douglis-Nirenberg elliptic systems, Cauchy-Kowalewski theorem.

(1) This paper is a condensed development of results obtained in the writer's doctoral dissertation at the University of Pennsylvania. 


$$
\begin{aligned}
D_{x} u^{2}+D_{y} u^{3} & =0 \\
D_{x} u^{1}-u^{2} & =0 \\
D_{y} u^{1}-u^{3} & =0 .
\end{aligned}
$$

The principal symbol of (1.1) is singular. Hence this system is not elliptic under the standard definition. However the system is elliptic in the generalized sense of Douglis-Nirenberg.

Now recall that if $y=0$ is an arbitrary but fixed hyperplane in real $\left(x_{1}, \cdots\right.$, $\left.x_{n}, y\right)$-space then any standard elliptic system of order $m$ can be written in the form

$$
F=L U=A D_{y}^{m} U+\sum_{i=1}^{n} B_{i} D_{x_{i}}^{m} U+\text { lower order terms, }
$$

where $D_{y}^{m} U, D_{x_{i}}^{m} U$ denote the vectors whose $j$ th components are $D_{y}^{\mu} U^{j}, D_{x_{i}}^{\mu_{j}} U^{j}$ respectively, $m=\max _{i} \mu_{i}$ and where $A$ is nonsingular in some neighborhood of $y=0$. Assume that the coefficient matrices $A, B_{i}$ are analytic in a neighborhood of $y=0$. Then solving (1.2) for the highest order normal derivatives $D_{y}^{m} U$ one can use the Cauchy-Kowalewski theorem to obtain an analytic solution $U$ of (1.2) with prescribed analytic Cauchy data

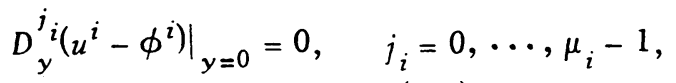

where the $\phi^{i}$ are analytic near $y=0$. Since (1.2) is linear the Holmgren theorem guarantees uniqueness among all solutions.

A glance at the Laplacian system in two variables mentioned above shows that writing the system (1.1) in the form (1.2), where $m=1$, yields a singular matrix $A$. That is, for Douglis-Nirenberg elliptic systems one cannot always solve for the highest (at least in the most naive sense) order normal derivatives in the system. In fact it will often happen that, for a particular system elliptic in our sense, every hyperplane is characteristic according to the standard definition of characteristic.

However, uniqueness and even existence for the usual Cauchy or initial value problem requires that the initial hyperplane be noncharacteristic. The alternative to having a free initial surface is to restrict the initial data by some compatibility conditions determined by the system. We formulate such conditions in $\$ 4$. These conditions are replaced in $\$ 6$ for a special subclass of our elliptic systems by a new condition which in some sense guarantees that every hyperplane is free with respect to the given system.

In addition, A. Pliś [9, p. 600] has discovered (Cohen independently found similar examples [3]) surprising examples of elliptic differential operators $L$ in $R^{n+1}$ with $C^{\infty}$ coefficients for which uniqueness for the Cauchy problem does not hold for $C^{\infty}$ Cauchy data. Hence we restrict our attention to the case of analytic data and analytic coefficients.

We will proceed as follows: 
In $\$ 2$ we fix notation and introduce the generalized notion of ellipticity due to Douglis and Nirenberg. Examples are used to show the diversity of this class of systems.

In $\$ 3$ we review some standard facts. In particular we note that all of our elliptic systems can be reduced to an equivalent system of first order which is also elliptic and that ellipticity is preserved under orthogonal changes of the independent variables.

In $\$ 4$ we formulate a "formal" Cauchy problem for our systems and introduce necessary and sufficient compatibility conditions for this problem to have a unique analytic solution. Here we are guided by Leray's work [6] on hyperbolic systems.

Existence and uniqueness for the formal Cauchy problem is proved in Theorems 1 and 2 respectively of $\$ 5$. The proof of existence uses techniques introduced by Morrey and Nirenberg [7, p. 280] for strongly elliptic systems. Uniqueness is shown to follow easily from existence.

In $\$ 6$ a second Cauchy problem is formulated for an important subclass of constant coefficient elliptic systems. This Cauchy problem in some sense eliminates the need for compatibility conditions and will occasionally be referred to as the "naive" Cauchy problem. Existence and uniqueness for this naive Cauchy problem are stated in Theorem 3. The proof makes use of Theorems 1 and 2.

We make several remarks in $\$ 7$. Most importantly we note that the systems considered in $\$ 6$ include all standard elliptic systems with constant coefficients. The obstacles to extending this theorem to a wider class of elliptic systems are briefly discussed.

The writer wishes to express his gratitude to Professor Jerry L. Kazdan for suggesting the central question of this paper and for his consistent encouragement and advice.

2. The systems to be considered. We will be concerned with functions $u(p)$ defined in domains in $R^{n+1}$, where $p=\left(x_{1}, \cdots, x_{n}, x_{n+1}\right)$. Denote $x_{n+1}$ by $y$ and $\left(x_{1}, \cdots, x_{n}\right)$ by $x$, so that $p=(x, y)$. We use the notation $D_{i}=\partial / \partial x_{i}, D=$ $\left(D_{1}, \cdots, D_{n+1}\right)$. If $a=\left(a_{1}, \cdots, a_{n+1}\right)$ we set $D_{1}^{a_{1}} \cdots D_{n+1}^{a_{n+1}}=D^{a}$. When we distinguish $y$ as the normal direction to a hyperplane we write $D_{y}$ in place of $D_{n+1}$. If $a=\left(a_{1}, \cdots, a_{n}, 0\right)$ we write $D_{x}^{a}$ rather than $D^{a}$ to emphasize that the operator involves only "tangential derivatives".

We treat systems of partial differential equations of the form

$$
\sum_{i=1}^{N} l_{i j}(p, D) u^{i}(p)=f_{i}(p), \quad i=1, \ldots, N,
$$

where the $l_{i j}(p, D)$ are polynomials in $D$ with coefficients depending analytically on $p$ over some domain $\mathfrak{D}$ in $R^{n+1}$. Following Douglis-Nirenberg we assume that two sets of integer weights $s_{1}, \cdots, s_{N} ; t_{1}, \cdots, t_{N}$ are associated $w$ ith the 
equations and the dependent variables $u^{j}$ respectively and that

$$
\operatorname{deg} l_{i j}(p, \xi) \leq s_{i}+t_{j}
$$

where deg refers to the degree of $l_{i j}$ as a polynomial in $\xi$. If $s_{i}+t_{j}<0$, then it is understood that $l_{i j} \equiv 0$.

Subtracting the constant $s=\max s_{i}$ from the system of weights $s_{1}, \cdots, s_{N}$ and adding the same constant to the system $t_{1}, \cdots, t_{N}$ one obtains new integer weights $s_{1}, \cdots, s_{N} ; i_{1}, \cdots, t_{N}$ also satisfying (2.2) but for which

$$
s_{i} \leq 0 ; \max _{i} s_{i}=0, \quad i=1, \ldots, N \text {. }
$$

Since for each fixed $j$ not all $l_{i j}$ vanish identically, we conclude that for some $i, s_{i}+t_{j} \geq 0$. This together with (2.3) gives

$$
0 \leq t_{j}, \quad j=1, \ldots, N \text {. }
$$

Definition. A system of the form (2.1) is elliptic in the sense of DouglisNirenberg if and only if

$$
L(p, \xi) \equiv \operatorname{det}\left(l_{i j}^{\prime}(p, \xi)\right) \neq 0 \quad \text { for real } \xi \neq 0,
$$

where $l_{i j}^{\prime}(p, \xi)$ consists of the terms in $l_{i j}(p, \xi)$ of exactly order $s_{i}+t_{j}$.

Condition $(2.5)$ is equivalent to requiring that $\operatorname{det}\left(l_{i j}(p, D)\right)$ be a standard elliptic operator of order $\sum_{i=0}^{N}\left(s_{i}+t_{i}\right)$.

The above definition of ellipticity is somewhat unsatisfactory in that it not at all obvious how to determine whether for a given system an appropriate set of integer weights can be found. Volevich [10, pp. 155ff.] introduced an easily verifiable definition of ellipticity which he showed to be equivalent to that given by Douglis-Nirenberg. He considers systems of the form (2.1). The determinant $A(p, \xi)=$ $\operatorname{det}\left(l_{i j}(p, \xi)\right)$ consists of sums and differences of terms of the form $l_{1, i_{1}} \ldots l_{N_{, i N}}$. Let $R$ be the maximal degree of such terms and let $r$ be the degree of $A(p, \xi)$, so that in general $r \leq R$. Then the system (2.1) is elliptic if and only if $r=R$ and $A^{\prime}(p, \xi) \neq 0$ for real $\xi \neq 0$, where $A^{\prime}(p, \xi)$ is the principal part of the polynomial $A(p, \xi)$, that is the part of degree $r$.

Remark 1. As Douglis-Nirenberg point out [4, p. 505] ellipticity as defined above may be destroyed by nonsingular transformations of the equations or the dependent variables. Hence, strictly speaking, a system is elliptic if and only if there are nonsingular transformations of the equations and the dependent variables which transform the system to one which is elliptic in the sense defined above.

Remark 2. Every standard elliptic system (1.2) is elliptic in the generalized sense just defined under the choice of integer weights $s_{i}=0, i=1, \cdots, N ; t_{i}=$ $\mu_{i}$, where $\mu_{i}$ are as in (1.2).

The following examples from [4] and [1] give some indication of the diversity of the systems included under our definition of ellipticity. 
Example 1. Recalling the first order system for the Laplacian (1.1) we note that according to the standard definition the characteristic determinant of $(1.1)$ is

$$
\left|\begin{array}{lll}
0 & \xi_{1} & \xi_{2} \\
\xi_{1} & 0 & 0 \\
\xi_{2} & 0 & 0
\end{array}\right|=0
$$

Hence this system is not elliptic in the standard sense. However, with the weights $s_{1}=0, s_{2}=s_{3}=-1$ assigned to the first, second and third equations of (1.1) respectively and the weights $t_{1}=2, t_{2}=1=t_{3}$ assigned to $u^{1}, u^{2}, u^{3}$ respectively, the characteristic determinant according to our definition becomes

$$
\left|\begin{array}{lll}
0 & \xi_{1} & \xi_{2} \\
\xi_{1} & -1 & 0 \\
\xi_{2} & 0 & -1
\end{array}\right|=\xi_{1}^{2}+\xi_{2}^{2}
$$

Consequently the system is elliptic in our generalized sense.

Example 2.

$$
\begin{aligned}
D_{x} u^{1}-D_{y}^{2} u^{2} & =f_{1}, \\
\left(D_{y}^{2}+a D_{y}\right) u^{1}+\left(D_{x}^{3}+D_{x y}\right) u^{2} & =f_{2} .
\end{aligned}
$$

Taking $s_{1}=0, s_{2}=1, t_{1}=1$ and $t_{2}=2$, the characteristic determinant has the form

$$
\left|\begin{array}{rr}
\xi_{1} & -\xi_{2}^{2} \\
\xi_{2}^{2} & \xi_{1}^{3}
\end{array}\right|=\xi_{1}^{4}+\xi_{2}^{4}
$$

Hence the system is elliptic in our sense, although not in the standard sense.

Note. Henceforth elliptic means Douglis-Nirenberg elliptic.

3. Some elementary lemmas. For elliptic systems of the form (2.1), conditions (2.3) and (2.4) can be shown [4, p. 506, Remark 2], to be no more general than

$$
0<t_{j}, \quad \text { for } j=1, \ldots, N ; \quad 0=s=\max _{i} s_{i}
$$

Consequently systems of integer weights associated with our elliptic system will always be assumed to satisfy (3.1).

Recall that in Example 1 of $\$ 2$ an elliptic system containing derivatives of order greater than 1 is reduced to an equivalent elliptic system which contains derivatives of at most order one. This is a special case of

Lemma 3.1 [1, p. 40]. Every elliptic system of the form (2.1) can be reduced to an equivalent elliptic system in which no derivatives of order greater than one appear.

The proof of Lemma 3.1 is given in [1]. We do not repeat it. However a rather detailed example of such a reduction is carried out in the proof of Theorem 1.

Remark. A generalized elliptic system of first order, that is one containing 
no derivatives of order greater than one, may have attached to it a system of weights $\left\{s_{i}\right\},\left\{t_{j}\right\}$ for which $s_{i_{0}}+t_{j_{0}}>1$ for some indices $i_{0}, j_{0}$. Example 1 of $\$_{2}$ illustrates this since there $s_{1}+t_{1}=2$.

One further preliminary is quite useful. As pointed out in the introduction we wish to study properties of solutions $U$ of elliptic systems of the form (2.1) satisfying certain initial conditions on some hyperplane or more generally on some analytic hypersurface in $R^{n+1}$. It will be convenient to introduce new coordinates $w_{1}, w_{2}, \cdots, w_{n+1}$ in $R^{n+1}$ which depend analytically on $x_{1}, \cdots, x_{n+1}$ and for which the initial hypersurface becomes the hyperplane $w_{n_{+1}}=0$. One can show that the new system obtained by rewriting (2.1) in terms of these new coordinates is itself elliptic. To be precise one has

Proposition 3.2. Given a system of the form (2.1) elliptic in a neighborbood $\Omega$ in $R^{n+1}$ and a point $p_{0} \in(\Omega \cap M)$, where $M$ is an analytic bypersurface in $R^{n+1}$, then there is an analytic diffeomorphism $S$ transforming $\Omega \cap M$ into the byperplane $x_{n+1}=0$, transforming $p_{0}$ to the origin and transforming the given system to another elliptic system of the form (2.1). Furthermore, if the original system bas analytic coefficients, then the transformed system also bas analytic coefficients.

Proof of Proposition 3.2. The proof is routine but lengthy. We omit it.

In $\$ \$ 4$ and 5 we make use of Proposition 3.2 to reduce the study of analytic Cauchy data on analytic hypersurfaces to the study of such data on hyperplanes.

4. The formal Cauchy problem. In this section we describe a "formal" Cauchy problem for elliptic systems of the form (2.1) which is a somewhat modified extension of the Cauchy problem discussed by Morrey and Nirenberg [7, p. 284] for strongly elliptic systems. In order to insure existence for this formal Cauchy problem we also introduce necessary compatibility requirements . (Morrey-Nirenberg [7] were not concerned with questions of existence and consequently compatibility conditions are not imposed there.) In formulating our compatibility conditions we were guided by those imposed by Leray [6, Pp. 206ff.] for his generalized Cauchy problem for generalized hyperbolic systems.

We shall seek solutions of (2.1) on domains of the form $G_{R} \cup \sigma_{R}$ where $G_{R}$ is the open hemiball

$$
G_{R}=\left\{(x, y) \in R^{n+1} \mid x_{1}^{2}+\cdots+x_{n}^{2}+y^{2}<R, y>0\right\}
$$

and

$$
\sigma_{R}=\left\{(x, y) \in R^{n+1} \mid x_{1}^{2}+\cdots+x_{n}^{2}<R, y=0\right\} .
$$


Recall that for an elliptic system of the form (2.1) the highest order derivatives of $u^{j}$ which appear are of order $\leq t_{j}$. This leads us to make the following

Definition. $U$ is a solution of the formal Cauchy problem for (2.1) in $G_{R} \cup \sigma_{R}$ with formal Cauchy data $\Phi=\left\{\phi^{j}\right\}$, where $\Phi$ is analytic in $G_{R} \cup \sigma_{R}$ if $U$ is a solution of (2.1) in $G_{R} \cup \sigma_{R}$ and satisfies

$$
\left.D_{y}^{k}{ }_{j}\left(u^{j}-\phi^{j}\right)\right|_{y=0}=0, \quad 0 \leq k_{j} \leq t_{j}-1, j=1, \ldots, N .
$$

Remark. This definition is not canonical since it is tied to the particular system of weights chosen and, as pointed out in [10], for a system of the form (2.1) there may be many systems of integer weights satisfying (2.2) and (3.1).

For arbitrary $\Phi(p)$, analytic near the origin, the formal Cauchy problem for (2.1) need not have a solution as is seen by returning to Example 1 of $\S 2$. We seek a solution of Example 1 satisfying the formal Cauchy conditions

$$
\begin{aligned}
& u^{1}-\phi^{1}=0, \quad D_{y}\left(u^{1}-\phi^{1}\right)=0, \\
& u^{2}-\phi^{2}=0, \quad u^{3}-\phi^{3}=0,
\end{aligned}
$$

Thus, for example, if $D_{y} \phi^{1}-\phi^{3} \neq 0$ on $y=0$ the problem has no solution. To guarantee existence it is necessary to introduce the following compatibility conditions.

Definition. If $\Phi(p)$ is analytic near the origin and if

$$
D_{y}^{K_{i}}\left(\sum_{i=1}^{N} l_{i j}(p, D) \phi^{j}(p)-f_{i}(p)\right)=0 ; \quad 0 \leq K_{i} \leq-s_{i}-1, i=1, \ldots, N,
$$

on $y=0$, where $\left(l_{i j}(p, D)\right)$ is as in $(2.1)$, then $\Phi$ is said to satisfy the formal compatibility conditions for Cauchy data for (2.1). In case $\left(-s_{i}-1\right)<0$, no assumption is made concerning the $i$ th equation in (2.1).

For Example 1 of $\$ 2$ considered above the compatibility requirement is that, on $y=0, \Phi$ satisfies

$$
D_{x} \phi^{1}-\phi^{2}=0, \quad D_{y} \phi^{1}-\phi^{3}=0, \quad \text { on } y=0 .
$$

Lemma 4.1. It suffices in solving the formal Caucby problem for elliptic sys. tems of the form (2.1) to consider only first order elliptic systems of the form (2.1).

Proof of lemma. Reduce the original elliptic system of the form (2.1) to a first order system via Lemma 3.1. The formal Cauchy data of the original system is then naturally reduced to formal Cauchy data for the equivalent first order sys. 
tem. This "reduced" formal Cauchy data can be seen to satisfy the formal compatibility conditions associated with the new first order system. Q.E.D.

5. Existence and uniqueness for the formal Cauchy problem. In this section we prove an existence theorem for the formal Cauchy problem discussed in $\$ 4$. Uniqueness then follows easily.

Theorem 1 (existence for the formal Cauchy problem). Let $\Phi$ be an analytic function of $p$ defined in some neigbborbood $\Omega$ of the origin in $R^{n+1}$ and satisfying the compatibility conditions (4.2) with respect to the system (2.1). Let (2.1) be elliptic in $G_{R} \cup \sigma_{R} \subset \Omega$. Then there exists a solution $U$ of (2.1) analytic in a neighborbood of the origin, $G_{R^{\prime}} \cup \sigma_{R^{\prime}}$, and satisfying on $y=0$ the formal Cauchy conditions (4.1).

Proof of Theorem 1. As pointed out in Lemma 4.1 it suffices to consider the case in which (2.1) is of first order. The basic idea of the proof is to replace (2.1) by a system which can be solved explicitly for the normal derivatives $D_{y}^{t}{ }^{j} u^{j}, j=$ $1, \cdots, N$. The proof consists of four steps, Steps 1 and 2 making use of techniques introduced in $[7, \mathrm{p} .280]$ for the case of strongly elliptic systems.

Step 1. Differentiate the $i$ th equation in $(2.1)\left(-s_{i}\right)$ times in the normal or $y$ direction. This gives the system of partial differential equations

$$
\sum_{j=1}^{N} \lambda_{i j}(p, D) u^{j}(p)=D_{y}^{-s} f_{i}(p), \quad i=1, \ldots, N,
$$

where

$$
\lambda_{i j}(p, D)=D_{y}^{-s}{ }_{i j}(p, D)
$$

From (5.2) it follows that

$$
\operatorname{deg} \lambda_{i j}(p, \xi) \leq s_{i}+t_{j}-s_{i}=t_{j}
$$

Step 2. Solve the system of equations (5.1) for the normal derivatives $D_{y}^{t} u^{j}$. To do so, first note that all solutions of (2.1) in $G_{R}$ are known to be analytic in $G_{R}$ (cf. $[7, \S 5]$ ). Hence in $G_{R}$ we may simplify (5.1) by interchanging the order of differentiation. This observation together with (5.3) and the fact that (2.1) is assumed to be of first order permit us to replace (5.1) for $p \in G_{R}$ by

$$
A(p)\left(D_{y}^{t}{ }^{j} u^{j}\right)+\sum_{|\beta| \leq 1 ;\left|a_{j}\right|<t} B_{a \beta^{\prime}} D_{x}^{\beta}\left(D_{y}{ }^{a}{ }^{j} u^{j}\right)=G(p),
$$


where $A, B_{\alpha \beta}$ are $N \times N$ matrices whose entries are real analytic functions of $p$ and where $G=\left(D_{y}^{-s_{i}} f_{i}\right)$.

To complete Step 2 we require

Lemma 5.1. For $p \in G_{R} \cup \sigma_{R}$, det $A(p) \neq 0$.

Proof of Lemma 5.1. The lemma is a direct consequence of (5.2) and the ellipticity of (5.1). Q.E.D.

Consequently for $p \in G_{R} \cup \sigma_{R}$ one can solve (5.4) and after simplifying obtain

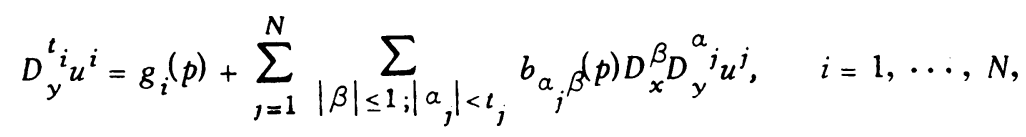

where $g_{i}$ and $b_{\alpha_{j} \beta}(p)$ are real analytic functions of $p, p \in G_{R} \cup \sigma_{R}$.

Step 3. Solve (5.5) for $U$ by reducing (5.5) to a first order system to which the Cauchy-Kowalewski theorem applies. To do so introduce the new unknown functions $u^{j, k_{j}}$ defined by

$$
u^{j, k}{ }_{j} \equiv D_{y}^{k} u^{j}, \quad \text { for } k_{j}=0, \cdots, t_{j}-1, j=1, \ldots, N \text {. }
$$

In this notation $u^{j} \equiv u^{j, 0}$. If we set $g^{i, 0} \equiv g_{i}$ and $g^{i, s} \equiv 0$ for $s>0$, then by replacing the normal derivatives in $(5.5)$ by the corresponding $u^{j, k_{j}}$ we obtain the first order system of equations

$$
\begin{aligned}
D_{y} u^{i, k_{i}}=g^{i, k_{i}}+\sum_{j=1}^{N} \sum_{k_{j}=0}^{t_{j}-1} \sum_{|\beta| \leq 1} b_{j, k_{j}} D_{x}^{\beta} u^{j, k_{j}} & \\
& \quad i, j=1, \ldots, N, k_{i}=0, \cdots, t_{i}-1,
\end{aligned}
$$

where again $g^{i, k_{i}}$ and $b_{j, k_{j}, \beta}$ are real analytic functions of $p$. Also in the new notation the Cauchy conditions (4.1) for (2.1) become

$$
\left.\left(u^{j, k_{j}}-D_{y}^{k_{j}} \phi^{j}\right)\right|_{y=0}=0 ; \quad j=1, \ldots, N, k_{j}=0, \cdots, t_{j}-1 .
$$

The Cauchy-Kowalewski theorem (cf. for example [5, pp. 28ff.]) applies to the system (5.7) with analytic initial data (5.8) to give a uniquely determined real analytic solution $\left\{v^{j, k_{j}}\right\}$ of (5.7)-(5.8) in a neighborhood $G_{R^{\prime}} \cup \sigma_{R^{\prime}}$ of the origin. The Cauchy-Kowalewski theorem does not rule out the existence of nonanalytic solutions. However, as we shall see later, none exist.

Step 4. The final step of the proof we state as

Lemma 5.2. Let $V=\left\{v^{j}\right\}=\left\{v^{j, 0}\right\}$. Then $V$ satisfies (2.1) on $G_{R^{\prime}} \cup \sigma_{R^{\prime}}$ and the Caucby conditions (4.1) on $\sigma_{R^{\prime}}$. 
Proof of Lemma 5.2. Since $\left\{v^{j, k_{j}}\right\}$ satisfies (5.7)-(5.8) on $G_{R^{\prime}} \cup \sigma_{R^{\prime}}$ it follows that $V$ satisfies (5.5) on $G_{R^{\prime}} \cup \sigma_{R^{\prime}}$ and (4.1) on $\sigma_{R^{\prime}}$. From the derivation of (5.5) one sees that $V$ satisfies (5.5) on $G_{R^{\prime}}$ if and only if $V$ satisfies (5.1) on $G_{R^{\prime}}$. Since $V$ satisfies (4.1) on $\sigma_{R^{\prime}}$ and since $\Phi$ satisfies (4.2) one obtains $V$ satisfies (5.1) on $\sigma_{R}, \cup G_{R}$, Q.E.D.

To complete the proof of the theorem recall that the $i$ th equation of (5.1) can be written as

$$
D_{y}^{-s}{ }_{i} \sum_{j=1}^{N} l_{i j}(p, D) u^{j}(p)=D_{y}^{-s}{ }_{i}(p)
$$

Integrating (5.9) with respect to $y$ and using the Cauchy data (4.1) and the compatibility conditions (4.2) to evaluate the resulting constants of integration, one sees that $V$ is a solution of (2.1). To verify this assertion it suffices to consider the first step in such an evaluation.

Assume for some $i, 1 \leq i \leq N$, that $-s_{i}>0$. Then for $y$ sufficiently small and for $p \in G_{R^{\prime}} \cup \sigma_{R^{\prime}}$,

$$
\int_{y=0}^{y=t} D_{y}^{-s} i \sum_{j=1}^{N} l_{i j}(p, D) v^{j}(p) d y=\int_{y=0}^{y=t} D_{y}^{-s} i f_{i}(p) d y .
$$

Consequently

$$
D_{y}^{-s_{i}-1} \sum_{j=1}^{N} l_{i j}(p, D) v^{j}(p)=D_{y}^{-s_{i}^{-1}} f_{i}(p)+q\left(x_{1}, \cdots, x_{n}\right) .
$$

Evaluate $q(x)$ by substituting $\left\{\phi^{j}\right\}$ from $(4.1)$ for $\left\{v^{j}\right\}$ on $y=0$ and recalling that (4.2) requires

$$
D_{y}^{-s_{i}-1} \sum_{j=1}^{N} l_{i j}(p, D) \phi^{j}=D_{y}^{-s_{i}^{-1}} f_{i} \text { on } y=0 \text {. }
$$

This gives $q\left(x_{1}, \cdots, x_{n}\right) \equiv 0$ and hence

$$
D_{y}^{-s_{i}-1} \sum_{j=1}^{N} l_{i j}(p, D) v^{j}=D_{y}^{-s_{i}^{-1}} f_{i}(p)
$$

Successive integration and evaluation of the constant of integration yields the desired result.

This completes the proof of Lemma 5.2 and of Theorem 1. Q.E.D. 
We can now easily resolve the question of uniqueness for the systems of Theorem 1. It follows from the derivation of (5.7) that any solution of (2.1) with the Cauchy data (4.1) satisfies (5.7) with the initial conditions (5.8). Consequently the existence of two distinct solutions of (2.1)-(4.1) would imply the existence of two distinct solutions of (5.7)-(5.8). This makes clear

Theorem 2 (uniqueness for the formal Cauchy problem). The formal Caucby problem (2.1)-(4.1) bas a unique solution $U \in C^{1}$. This solution is analytic in a neighborbood of the origin.

Proof of Theorem 2. The Holmgren uniqueness theorem (cf., e.g., [5]) applies directly to (5.7)-(5.8) to give uniqueness for (2.1)-(4.1). Q.E.D.

We can now give a partial answer to the question in the Introduction.

Corollary 5.3. Suppose $U \in C^{1}$ is a solution of the elliptic system (2.1) satisfying (4.1) for $\Phi \equiv 0$. Then $U \equiv 0$ in some neighborbood of the origin.

Proof of Corollary 5.3. One can easily show that $\Phi=0$ satisfies (4.2). Hence we can apply Theorems 1 and 2 to conclude that $U$ is the unique analytic solution of (2.1) satisfying (4.1) for $\Phi=0$. But from (5.5) in the proof of Theorem 1 it is clear that all the Taylor coefficients of $U$ must vanish on $y=0$. Since $U$ is analytic, $U \equiv 0$ in a neighborhood of the origin. Q.E.D.

6. The "naive" Cauchy problem. In this section we restrict our attention to first order constant coefficient elliptic systems satisfying a further condition which is described below. For these systems data will be specified only on hyperplanes and not on more general hypersurfaces. The main result is stated in Theorem 3. In $\$ 7$ it is shown that the systems considered in this section include the standard elliptic systems.

We consider a constant coefficient elliptic system of first order of the form (2.1) with weights $s_{i},{ }_{j}$ satisfying the usual conditions (3.1). Since (2.1) is assumed to be of first order it can be written as

$$
\left(A D_{y}+\sum_{i=1}^{n} \widetilde{B}_{i} \dot{D}_{i}+\widetilde{C}\right) U=\widetilde{F}
$$

where $\tilde{F}=\left(f_{1}(p), \cdots, f_{N}(p)\right)$ and $A, \widetilde{B}$ and $\tilde{C}$ and $N \times N$ matrices with real constant entries. The constant coefficient elliptic systems of the form (6.1) with which we are concerned in this section satisfy the further condition

$$
\text { Rank of } A=\sum_{i=1}^{N}\left(s_{i}+t_{i}\right) \equiv \mu \text {. }
$$


Remark. It is clear from (5.7) and (6.1) that in general rank of $A \geq \mu$. We replace $(6.1)$ by a more tractable system in

Proposition 6.1. There exists a nonsingular $N \times N$ constant matrix $T$ sucb that if $V=T^{-1} U$ then $U$ satisfies (6.1) if and only if $V$ satisfies the system of equations

$$
D_{y} \bar{V}=H\left(D_{x}\right) \bar{V}+\bar{F}
$$

$$
(6.3)^{\perp} \quad V^{\perp}=G\left(D_{x}\right) \bar{V}+I,
$$

where $\bar{V}=\left(v^{1}, \cdots, v^{\mu}\right), V^{\perp}=\left(v^{\mu+1}, \cdots, v^{N}\right), H$ and $G$ are $\mu \times \mu$ and $(N-\mu) \times \mu$ dimensional matrices respectively whose entries are polynomials, possibly of degree greater than one, in the tangential derivatives $D_{x}$, and where $I(p)$ is an appropriate real analytic function of $p$.

The proof of Proposition 6.1 is given at the end of this section immediately following the proof of Corollary 6.3.

We can now make the following

Definition. $U$ is a solution of the "naive" Caucby problem for (6.1) with Cauchy data $\Psi$ on $y=0$ if $V$ satisfies (6.3) and if $\left.(\bar{V}-\bar{\Phi})\right|_{y=0}=0$, where $\Psi=$ $T \Phi$ and $\bar{\Phi}=\left(\phi^{1}, \cdots, \phi^{\mu}\right)$.

The next lemma shows that all the Taylor coefficients on $y=0$ of a solution of (6.1) are uniquely determined by its naive Cauchy data.

Lemma 6.2. If $\bar{V}=\bar{\Phi}$ on $y=0$ and if

$$
\Phi^{\perp}=G\left(D_{x}\right) \bar{\Phi}+I \text { on } y=0,
$$

where $\Phi$ is an arbitrary real function of $p$ analytic near the origin, $\Phi^{\perp}=\left(\phi^{\mu+1}, \cdots, \phi^{N}\right)$ and $G, I$ are from $(6.3)^{\perp}$, then

$$
D^{a} V=D^{a} \Phi \text { on } y=0 \text {, for all } a .
$$

Proof of Lemma 6.2. First, since $\bar{\Phi}=\bar{V}$ on $y=0$ one obtains that $D_{x}^{\beta} \bar{\Phi}=$ $D_{x}^{\beta} \bar{V}$ for all $\beta$. If $D^{a}=D_{x}^{\beta} D_{y}^{a_{n+1}}$ it follows from $\left(\overline{6.3)}\right.$ by induction on $\left|\alpha_{n+1}\right|$ that on $y=0, D^{a} \bar{\Phi}=D^{a} \bar{V}$ for all $\alpha$. Similarly one obtains from (6.4) by induction on $\left|a_{n+1}\right|$ that $D^{a} \Phi^{\perp}=D^{a} V^{\perp}$ on $y=0$, for all $a$. Q.E.D.

The main result of this section is

Theorem 3. Let $\Phi$ be as in Lemma 6.2. If (6.1) satisfies condition (6.2) then in some neighborbood of the origin there exists a unique (among all $C^{1}$ functions) analytic solution $U$ of (6.1) satisfying $U=\Psi$ on $y=0$ where $\Psi \equiv T \Phi, T$ as in Proposition 6.1. 
Proof of Theorem 3. Use Lemma 6.2 to verify that, for (6.1) rewritten in the form (2.1), $\Psi$ satisfies the compatibility conditions (4.2). The theorem then follows from Theorems 1 and 2. Q.E.D.

Theorem 3 permits us to give another partial answer to the question of $\$ 1$.

Corollary.6.3. If $U \in C^{1}$ is a solution of (6.1) and if $U=0$ on $y=0$, then $U \equiv 0$ in a neighborbood of the origin.

Proof of Corollary 6.3. To apply Theorem 3 verify that, for $\Psi=0, \Phi=T^{-1} \Psi$ satisfies (6.4). Q.E.D.

It remains to give the

Proof of Proposition 6.1. Condition (6.2) implies that (6.1) can be written in the form

$$
\pi_{\mu} D_{y} V+\sum_{i=1}^{n} B_{i} D_{i} V+C V=F
$$

where $\pi_{\mu}$ is defined by $\pi_{\mu}\left(a_{1}, \cdots, a_{N}\right) \equiv\left(a_{1}, \cdots, a_{\mu}, 0, \cdots, 0\right)$. To obtain (6.3) separate the first $\mu$ equations and the last $(N-\mu)$ equations of $(6.6)$ by writing

$$
\overline{(6.7)} D_{y} \bar{V}+\sum_{i=1}^{n} \Gamma_{i} D_{i} \bar{V}+\sum_{i=1}^{n} \Gamma_{i}^{\perp} D_{i} V^{\perp}+M \bar{V}+M^{\perp} V^{\perp}=\bar{F}
$$

$$
(6.7)^{\perp}\left(\sum_{i=1}^{N} \beta_{i} D_{i}+\mathcal{C}\right) \bar{V}+\left(\sum_{i=1}^{n} \beta_{i}^{\perp} D_{i}+\mathcal{C}^{\perp}\right) V=F^{\perp}
$$

where the matrices $\beta_{i}, \beta_{i}^{\perp}, \Gamma_{i}, \Gamma_{i}^{\perp}, \mathcal{C}, \mathcal{C}^{\perp}, M, M^{\perp}$ are defined schematically as

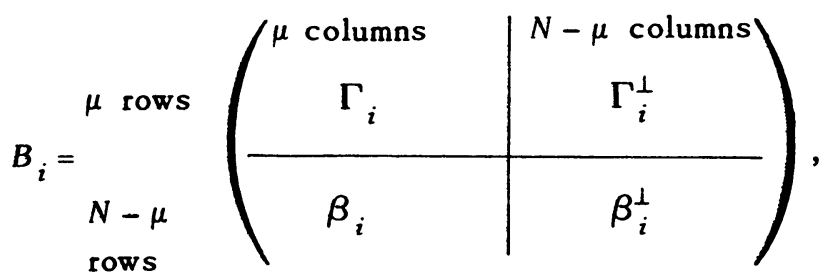

$$
C=\underset{\substack{\mu \text { columns } \\
M-\mu \\
\text { rows }}}{\mu \text { rows }}\left(\begin{array}{c}
N-\mu \text { columns } \\
M^{\perp} \\
\hline
\end{array}\right)
$$

$B_{i}$ and $C$ as in (6.6). 
To complete the proof of Proposition 6.1 solve $(6.7)^{\perp}$ for $V^{\perp}$ in terms of tangential derivatives of $\bar{V}$ and of $F^{\perp}$ and substitute the result in (6.7). To do so, however, requires

Lemma 6.5. In $(6.7)^{\perp}, \operatorname{det}\left(\sum_{i=1}^{n} \beta_{i}^{\perp} \xi_{i}+C^{\perp}\right)=a \neq 0$, where $a$ is a constant.

Proof of Lemma 6.5. The proof follows from the ellipticity of (6.1) and a tedious though routine computation of the required determinant. This completes the proof of Lemma 6.5 and of Proposition 6.1. Q.E.D.

7. Remarks. A. The systems of Theorem 3, that is, those of the form (6.1) which satisfy (6.2) are quite a broad class. In particular every standard elliptic system (described in $\$ 1$ ) can be reduced to a first order system of the form (6.1) satisfying (6.2).

The procedure is precisely that used in Lemma 3.1 except for the following difference. We restrict attention to $C^{K}$ solutions where $K$ is the order of the standard elliptic system under consideration. Then we may interchange the order of differentiation in the system at will. In particular if a term of the form $D^{\alpha}$ appears in $l_{i j}(D)$ we rewrite it as $D_{x}^{a^{\prime}} D_{y}^{\beta},\left|\alpha^{\prime}\right|+|\beta|=|\alpha|$.

Otherwise the procedure is identical to that of Lemma 3.1. The resulting first order system can be shown to be of the form (6.1) with rank $A=\sum_{i=1}^{N}\left(s_{i}+t_{i}\right)$.

$B$. The writer knows of no nontrivial example of a system of the form (6.1) which fails to satisfy (6.2). However, trivial examples such as the following abound.

$$
\begin{aligned}
D_{x} u^{2}+D_{y} u^{3} & =0 \\
D_{x} u^{1}-u^{2} & =0 \\
D_{y} u^{1}-u^{3} & =0 \\
D_{y} u^{2}+u^{4} & =0
\end{aligned}
$$

with the weights $t_{1}=2, t_{2}=1, t_{3}=1, t_{4}=0, s_{1}=0, s_{2}=-1, s_{3}=-1, s_{4}=0$. If (7.1) is written in the form $(6.1)$ it is seen that rank $A=3 \neq 2=\sum_{i=1}^{N}\left(s_{i}+t_{i}\right)$. However, the unknown $u^{4}$ can be eliminated in (7.1) and written explicitly in terms of the other unknown functions $u^{1}, u^{2}, u^{3}$ and the ir derivatives, viz., $u^{4}=D_{y} u^{2}$. Now the reduced system consisting of the first three equations of (7.1) does satisfy (6.2). Consequently Theorem 3 applies to these equations, that is, specifying $\bar{V}=\left\{u^{1}, u^{3}\right\}$ on $y=0$ determines a unique analytic solution $U$ of (7.1).

It would be of interest to find a nontrivial example of the type discussed and to resolve Theorem 3 in this case. 
C. For analytic coefficient systems of the form (6.1) the proof of Theorem 3 breaks down at several points. In particular the analog of Lemma 6.5 does not hold in this case. Thus it remains open as to whether Theorem 3 can be extended to analytic systems of the form (6.1) which satisfy (6.2).

\section{BIBLIOGRAPHY}

1. S. Agmon, A. Douglis and L. Nirenberg, Estimates near the boundary for solutions of elliptic partial differential equations satisfying general boundary conditions. II, Comm. Pure Appl. Math. 17 (1964), 35-92. MR 28 \#5252.

2. F. Browder, The zeros of solutions of elliptic partial differential equations with analytic coefficients, Arch. Math. (Basel) 19 (1968), 183-187. MR 38 \#397.

3. P. Cohen, The non-uniqueness of the Cauchy problem, ONR Technical Report \#93, Stanford University, Stanford, Calif., 1960.

4. A. Douglis and L. Nirenberg, Interior estimates for elliptic systems of partial differential equations, Comm. Pure Appl. Math. 8 (1955), 508-538.

5. P. D. Lax, Partial differential equations, Appendix by A. Douglis, Lecture Notes, New York University, 1951.

6. J. Leray, Hyperbolic differential equations, Institute for Advanced Study, Princeton, N. J., 1953. MR 16, 139.

7. C. B. Morrey and L. Nirenberg, On the analyticity of the solutions of linear elliptic systems of partial differential equations, Comm. Pure Appl. Math. 10 (1957), 271-290. MR 19, 654 .

8. S. Perlis, Theory of matrices, Addison-Wesley, Reading, Mass., 1952. MR 14, 6.

9. A. Pliś, A smooth linear elliptic differential equation without any solution in a sphere, Comm. Pure Appl. Math. 14 (1961), 599-617. MR 25 \#307.

10. L. R. Volevič, A problem in linear programming stemming from differential equa. tions, Uspehi Mat. Nauk 18 (1963), no. 3 (111), 155-162. (Russian) MR 28 \#5248.

DEPARTMENT OF MATHEMATICS, PENNSYLVANIA STATE UNIVERSITY, UNIVERSITY

PARK, PENNSYLVANIA 16802 